\title{
The Dynamics of Wealth Inequality under Endogenous Fertility: A Remark on the Barro-Becker Model with Heterogenous Endowments
}

\author{
Stefano Bosi ${ }^{1}$, Raouf Boucekkine ${ }^{2,3}$, Thomas Seegmuller ${ }^{4}$ \\ ${ }^{1}$ THEMA and University of Cergy-Pontoise, Cergy, France \\ ${ }^{2}$ IRES and CORE, Catholic University of Louvain, Louvain-La-Neuve, Belgium \\ ${ }^{3}$ GREQAM, Aix-Marseille University, Marseille, France \\ ${ }^{4}$ CNRS and GREQAM, Paris, France \\ E-mail: stefano.bosi@u-cergy.fr, \{raouf.boucekkine, thomas.seegmuller\}@univmed.fr \\ Received April 13, 2011; revised April 24, 2011; accepted April 27, 2011
}

\begin{abstract}
Implicit in the seminal contribution of Barro-Becker [1], the lack of persistence of inequality in the presence of endogenous fertility is one of the most striking features of the models à la Barro-Becker. In this pedagogical note, we show how to uncover and interpret the latter property using standard optimization in contrast to the dynamic programming under homogeneity usually invoked in this literature.
\end{abstract}

Keywords: Endogenous Fertility, Heterogeneous Households, Optimal Growth

\section{Introduction}

Following the paper by Chatterjee [2], several contributions have introduced households' heterogeneity in the Ramsey model through wealth inequality. The main focus of this recent literature is the existence of a representative consumer summarizing the average behavior of the economy and the persistence of inequalities during the transitional dynamics and in the steady state.

These questions have been extensively studied by Chatterjee, Caselli and Ventura and Garcia- Peñalosa and Turnovsky [2-4] in the standard setup where households derive utility from consumption only. These papers show that while wealth inequalities may be reduced, they do persist in the long-run. In contrast, another much thinner literature introducing endogenous fertility in line with Barro and Becker [1,5] has shown that inequalities do not persist. Implicit in these seminal papers, this striking property has been demonstrated in a quite general model using dynamic programming with homogenous functions by Alvarez [6]. Precisely because it is a general approach and because it does not rely on easily interpretable Euler equations, the latter framework is not reader-friendly. This note makes use of standard optimization and the resulting Euler equations to provide a simple and intuitive reading of the lack of inequality persistence in the Barro-Becker models. More concretely, we show that when households smooth consumption over time and optimally choose the number of children, individual consumptions should be equal after one period. This striking property essentially derives from the resulting equality between the marginal benefit and cost of bequest: consumptions are then shown to be independent of the capital distribution after one period. This in turn implies that fertility rates and capital held by each household become also identical after one period. Therefore, introducing endogenous fertility in the optimal growth model rules out wealth inequalities after only one adjustment period. Finally, it is important to notice that we show our result for a given sequence of prices over time. This means that it does not depend on the specification of the production sector, and occurs under exogenous as well as endogenous growth.

This note is organized as follows. In the next section, we present the behavior of heterogeneous households. In Section 3, we show our result on the loss of heterogeneity after one period, while some technical details are reported in the Appendix.

\section{Households’ Behavior}

We extend the Barro and Becker model [5] to account for wealth heterogeneity. We consider an eco- nomy with $H$ dynasties of altruistic households, $i=1, \cdots, H$, that 
differ in their individual wealth. In other words, we focus on heterogeneity in initial capital endowment $k_{i 0}$, i.e. $k_{h 0} \neq k_{j 0}$ for $h \neq j$.

As in the seminal setting, each person is assumed to live for two periods, childhood and adulthood, and has children at the beginning of his adult period. Parents are altruistic towards their children, i.e. utility depends on their own consumption, the number of surviving children and the utility of each child. The utility of an adult of type $i$ belonging to the generation born at $t-1$, is given by:

$$
U_{i t}=u\left(c_{i t}\right)+\left(\alpha n_{i t}^{-\varepsilon}\right) n_{i t} U_{i t+1}
$$

where $c_{i t}$ is the individual consumption giving an instantaneous utility $u\left(c_{i t}\right)$, while $n_{i t}$ is the number of children. We notice that $\alpha n_{i t}^{-\varepsilon}$ measures the degree of altruism towards each child, with $\alpha, \varepsilon \in(0,1)$, and $U_{i t+1}$ is the utility attained by each child. We further assume:

Assumption 1 The utility function $u\left(c_{i}\right)$ is defined on $R_{+}$, two-times continuously differentiable on $R_{++}$, strictly increasing $\left(u^{\prime}\left(c_{i}\right)>0\right)$, strictly concave

$\left(u^{\prime \prime}\left(c_{i}\right)<0\right)$ and satisfies $-\frac{u^{\prime \prime}\left(c_{i}\right) u\left(c_{i}\right)}{u^{\prime}\left(c_{i}\right)^{2}}>\frac{\varepsilon}{1-\varepsilon} .{ }^{1}$ Noting $\sigma\left(c_{i}\right)=c_{i} u^{\prime}\left(c_{i}\right) / u\left(c_{i}\right)$, we assume $0<\sigma\left(c_{i}\right)<1-\varepsilon$ and $\sigma\left(c_{i}\right)$ is non-increasing.

Notice that the utility function $u\left(c_{i}\right)=c_{i}^{\sigma}$, with $\sigma<1-\varepsilon$, satisfies Assumption 1. ${ }^{2}$

As stressed by Becker and Barro [1], the recursive model (1) can be equivalently written as an optimal growth model where the household of type $i$ maximizes a dynastic utility:

$$
\sum_{t=0}^{\infty} \alpha^{t} N_{i t}^{1-\varepsilon} u\left(c_{i t}\right)
$$

with $N_{i t}$ the size of the $i$ th subpopulation at period $t$, under a sequence of budget constraints:

$$
c_{i t}+n_{i t} k_{i t+1}=R_{t} k_{i t}+w_{t}\left(1-\beta n_{i t}\right)
$$

$t=0,1, \cdots$, and given the unequal distribution of initial capital $k_{i 0}$, with $k_{h 0} \neq k_{j 0}$ for $h \neq j$.

The left-hand side of (3) represents households' expenditures. In particular, $k_{i t+1}$ represents the bequest per child, through physical as well as human capital used for production in the next period. The right-hand side of (3) represents the disposable income, where $w_{t}$ is the wage rate and $R_{t} \equiv 1-\delta+r_{t}$ the gross return on capital, with $\delta \in(0,1)$ the depreciation rate of capital and $r_{t}$ the

\footnotetext{
${ }^{1}$ As it is explicitly shown in Becker and Barro [7], this inequality ensures that the second order conditions are satisfied for the house- holds' utility maximization.

${ }^{2}$ This utility function is considered in the seminal contribution of and Barro and Becker [5].
}

real interest rate. As mentioned in the introduction, we do not specify the production sector in order to highlight that our result holds irrespective of the production technology. Accordingly, we assume that the sequence of prices $\left(w_{t}, R_{t}\right), t=0,1, \cdots$, is given.

At adulthood, each household is endowed with one unit of time that she shares between labor and leisure. The time cost of rearing children is given by $\beta n_{i t} w_{t}$, where $\beta$ is the constant cost per child in units of time. Leisure time $\beta n_{i t}=1-l_{i t} \in(0,1)$ is spent with children, whereas $l_{i t}$ is the individual labor supply at period $t$.

Since $n_{i t}$ represents the population growth factor of dynasty $i$, the size of a dynasty at time $t$ is given by:

$$
N_{i t}=n_{i t-1} N_{i t-1}=N_{i 0} \prod_{s=0}^{t-1} n_{i s}
$$

where $N_{i 0}>0$ are given for $i=1, \cdots, H$, and heterogeneous initial population sizes are not excluded, i.e. $N_{h 0} \neq N_{j 0}$ for $h \neq j$.

Maximizing utility (2) under the budget constraints (3), the household $i$ chooses a sequence $\left(k_{i t+1}, n_{i t}, c_{i t}\right)_{t=0}^{\infty}$ of saving, consumption, number of children. The household behavior may be summarized by: ${ }^{3}$

$$
\begin{gathered}
R_{t} k_{i t}+w_{t}\left(1-\beta n_{i t}\right)=c_{i t}+n_{i t} k_{i t+1} \\
u^{\prime}\left(c_{i t}\right) / u^{\prime}\left(c_{i t+1}\right)=\alpha n_{i t}^{-\varepsilon} R_{t+1} \\
\alpha^{t} N_{i t}^{1-\varepsilon} u^{\prime}\left(c_{i t}\right)\left(\beta w_{t}+k_{i t+1}\right)=\frac{1-\varepsilon}{n_{i t}} \sum_{s=t+1}^{\infty} \alpha^{s} N_{i s}^{1-\varepsilon} u\left(c_{i s}\right)
\end{gathered}
$$

with the transversality condition:

$$
\lim _{t \rightarrow+\infty} \alpha^{t} N_{i t}^{1-\varepsilon} u^{\prime}\left(c_{i t}\right) n_{i t} k_{i t+1}=0
$$

Equation (5) is the household's budget constraint. The intertemporal trade-offs are summarized by (6) and (7). The Euler Equation (6) shows how dynasties smooth consumption over time. Since the marginal utility of consumption depends on population size $N_{i t}$ and total bequest $n_{i t} k_{i t+1}$, which in turn depend on the number of children, this choice between current and future consumption depends on the fertility rate. Finally, Equation (7) determines the optimal number of children. The cost per child in unit of time and in terms of bequest (on the left-hand side) is equal to the discounted sum of the marginal utility gains over all the subsequent periods (on the right-hand side).

\section{The "Loss" of Heterogeneity}

Using the optimal behavior of the households, we show now that from period 1 onwards, the individual consumptions $c_{i t}$, the number of children $n_{i t}$ and the wealth

${ }^{3}$ See the Appendix for details. 
$k_{i t}$ become equal. As underlined above, this property is demonstrated without specifying the production sector, the sequence of prices $\left(w_{t}, R_{t}\right)$, for $t=0,1, \cdots$, is given.

As explained in the Appendix, Equation (7) can also be written as:

$$
\begin{aligned}
& (1-\varepsilon) \alpha u\left(c_{i t+1}\right)+\alpha u^{\prime}\left(c_{i t+1}\right)\left(R_{t+1} k_{i t+1}+w_{t+1}-c_{i t+1}\right) \\
& =n_{i t}^{\varepsilon} u^{\prime}\left(c_{i t}\right)\left(\beta w_{t}+k_{i t+1}\right)
\end{aligned}
$$

Substituting Equation (6), we get:

$$
\begin{aligned}
& c_{i t+1} \frac{1-\varepsilon-\sigma\left(c_{i t+1}\right)}{\sigma\left(c_{i t+1}\right)}=\beta w_{t} R_{t+1}-w_{t+1}, \\
& \text { for } t=0,1, \cdots, \text { and } i=1, \cdots, H
\end{aligned}
$$

Under Assumption 1, there is a unique solution $c_{i t+1}$ solving this equation. In other words, for all $t=1, \cdots$ and $i=1, \cdots, H$, we have $c_{i t} \equiv c_{t}$, with

$$
c_{t}=\left(\beta w_{t-1} R_{t}-w_{t}\right) \frac{\sigma\left(c_{t}\right)}{1-\varepsilon-\sigma\left(c_{t}\right)}
$$

From period $t=1$ onwards, the individual consumptions are equal. One can see that $c_{t}$ is increasing in the time cost per child measured in physical units of the next period, because the dynasty substitutes investment in children for future consumption. However, less children means a lower labor force, which explains that consumption is decreasing in the current wage.

Most importantly for our purpose, individual consumptions get equalized because $c_{i t}$ does not depend on capital holding $k_{i t}$ and, therefore, it is independent of the distribution of capital. Indeed, recall that $k_{i t+1}$ represents the bequest per child given by a parent living at period $t$. Equation (8), represents the intertemporal trade-off to have children: it equalizes the marginal benefit to have children (on the left-hand side) to its marginal cost (on the right-hand side). In particular, $\alpha u^{\prime}\left(c_{i t+1}\right) R_{t+1} k_{i t+1}$ represents the marginal benefit of bequest, while $n_{i t}^{\varepsilon} u^{\prime}\left(c_{i t}\right) k_{i t+1}$ is its marginal cost. Taking into account the optimal choice between current and future consumption (see (6)), the marginal benefit of bequest becomes equal to its marginal cost, implying individual consumption to be independent of wealth.

Since consumptions are identical from $t=1$, the Euler Equation (6) implies that the fertility rates are also identical from $t=1$, i.e. $n_{i t} \equiv n_{t}$ for all $t=1, \cdots$ and $i=1, \cdots, H$. Using (10), we obtain:

$$
n_{t}^{\varepsilon}=\alpha R_{t+1} u^{\prime}\left(c_{t+1}\right) / u^{\prime}\left(c_{t}\right)
$$

Because consumptions do not depend on the wealth distribution and get equalized across dynasties, the number of child is also equalized for $t=1, \cdots$.
A question now emerges. What can we infer for individual wealth? We show that capital distribution becomes also homogenous from period $t=1$.

Proposition 1 Under Assumption 1, for $t=1, \cdots$, the distribution of capital is also homogeneous, i.e. $k_{i t}=k_{t}$ for all $i=1, \cdots, H$.

Proof. Using (5) and (7), we get:

$$
R_{t} k_{i t}-c_{i t}+w_{t}=\frac{1-\varepsilon}{u^{\prime}\left(c_{i t}\right)} \sum_{s=t+1}^{\infty} \alpha^{s-t}\left(\frac{N_{i s}}{N_{i t}}\right)^{1-\varepsilon} u\left(c_{i s}\right)
$$

We have $c_{i t}=c_{t}$ and $n_{i t}=n_{t}$ for all $t=1, \cdots$, and $N_{i s}=N_{i 1} \prod_{t=1}^{s-1} n_{t}$. Therefore, Equation (12) writes for $t=1$ :

$$
R_{1} k_{i 1}+w_{1}-c_{1}=\frac{1-\varepsilon}{u^{\prime}\left(c_{1}\right)} \sum_{s=2}^{\infty} \alpha^{s-1}\left(\prod_{t=1}^{s-1} n_{t}\right)^{1-\varepsilon} u\left(c_{s}\right)
$$

This shows that the capital distribution is homogeneous at $t=1$, i.e. $k_{i 1} \equiv k_{1}$ for all $i=1, \cdots, H$. From $t=1$, the budget constraint (5) becomes:

$$
k_{i t+1}=\frac{R_{t}}{n_{t}} k_{i t}+\frac{w_{t}\left(1-\beta n_{t}\right)-c_{t}}{n_{t}}
$$

Since $k_{i 1}=k_{j 1}=k_{1}$ for all $i, j=1, \cdots, H$, we deduce that $k_{i t}=k_{j t} \equiv k_{t}$ for all $i, j=1, \cdots, H$ and $t=1, \cdots$ This proves that the capital distribution is equal from $t=1$ onwards.

This proposition shows that introducing endogenous fertility in the optimal growth model with heterogeneous households has critical implications for the capital distribution. In contrast to previous contributions with exogenous population size starting with Chatterjee [2], inequality does not persist and the wealth distribution becomes homogenous from $t=1$. As already emphasized, this arises from the disjunction between individual consumptions and capital holdings, which is explained by the fact that households do not only make an intertemporal choice between present and future consumptions, but also optimally choose the number of their children.

We now come to some observations on the robustness of our findings.

Remark 1. In our analysis, we assume that the counterpart of having children is a time cost per child $\beta w_{t}$. Another traditional cost specification is to consider a cost in terms of the final good, i.e. $\tilde{\beta}=\beta w_{t}[1,5]$. One may easily see that this alternative specification of the cost per child does not change our conclusions on the loss of heterogeneity from $t=1$.

Remark 2. As already underlined, our result is shown for a given sequence of prices $\left(w_{t}, R_{t}\right), t=0, \cdots$, This means that it neither depends on the microeconomic 
foundations of the production sector, nor on the assumptions on the technology. As a direct implication, if one introduces a standard convex technology with constant returns, the economy may obviously converge to a steady state with a homogenous wealth distribution. If one introduces a technology leading to endogenous growth [8], there may exist a unique balanced growth path where all the individuals have the same wealth growing at a similar well-defined rate.

\section{References}

[1] F. Alvarez, "Social Mobility: The Barro-Becker Children Meet the Laitner-Loury Dynasties," Review of Economic Dynamics, Vol. 2, No. 1, 1999, pp. 65-103. doi:10.1006/redy.1998.0052

[2] R. J. Barro and G. S. Becker, "Fertility Choice in a Model of Economic Growth,” Econometrica, Vol. 57, No. 2, 1989, pp. 481-501. doi:10.2307/1912563

[3] G. S. Becker and R. J. Barro, "A Reformulation of the Economic Theory of Fertility," Quarterly Journal of Economics, Vol. 103, No. 1, 1988, pp. 1-25. doi:10.2307/1882640
[4] G. S. Becker and R. J. Barro "A Reformulation of the Economic Theory of Fertility,” The Quarterly Journal of Economics, Vol. 103, No. 1, 1988, pp. 1-25. doi: $10.2307 / 1882640$

[5] S. Bosi and T. Seegmuller, "Mortality Differential and Growth: What Do We Learn from the Barro-Becker Model?” Mathematical Population Studies, Forthcoming, 2010.

[6] F. Caselli and J. Ventura, "A Representative Consumer Theory of Distribution," American Economic Review, Vol. 90, No. 4, 2000, pp. 909-926. doi:10.1257/aer.90.4.909

[7] S. Chatterjee, "Transitional Dynamics and the Distribution of Wealth in a Neoclassical Growth Model,” Journal of Public Economics, Vol. 54, No. 1, 1994, pp. 97-119. doi:10.1016/0047-2727(94)90072-8

[8] C. Garcia-Peñalosa and S. J. Turnovsky, "The Dynamics of Wealth Inequality in a Simple Ramsey Model: A Note on the Role of Production Flexibility," Macroeconomic Dynamics, Vol. 13, No. 2, 2009, pp. 250-262. doi:10.1017/S1365100508070508 


\section{Appendix}

\section{The optimal behavior of household}

We derive the infinite-horizon Lagrangian function with respect to $c_{i t}, k_{i t}, n_{i t}$ :

$$
\begin{aligned}
& \sum_{t=0}^{\infty} \alpha^{t} N_{i t}^{1-\varepsilon} u\left(c_{i t}\right) \\
& +\sum_{t=0}^{\infty} \lambda_{i t}\left[R_{t} k_{i t}+w_{t}\left(1-\beta n_{i t}\right)-c_{i t}-n_{i t} k_{i t+1}\right]
\end{aligned}
$$

in order to obtain the first-order conditions:

$$
\begin{gathered}
\lambda_{i t}=\alpha^{t} N_{i t}^{1-\varepsilon} u^{\prime}\left(c_{i t}\right) \\
\lambda_{i t} n_{i t}=\lambda_{i t+1} R_{t+1} \\
\lambda_{i t}\left(\beta w_{t}+k_{i t+1}\right)=\frac{1-\varepsilon}{n_{i t}} \sum_{s=t+1}^{\infty} \alpha^{s} N_{i s}^{1-\varepsilon} u\left(c_{i s}\right)
\end{gathered}
$$

with the transversality condition:

$$
\lim _{t \rightarrow+\infty} \alpha^{t} N_{i t}^{1-\varepsilon} u^{\prime}\left(c_{i t}\right) n_{i t} k_{i t+1}=0
$$

Noticing that $n_{i t}=N_{i t+1} / N_{i t}$, we get from (16) and (17) a sequence of Euler Equations (6). Using (16) and
(18), we obtain (7). From (18), we also have:

$$
\begin{aligned}
& n_{i t} \lambda_{i t}\left(\beta w_{t}+k_{i t+1}\right) \\
& =(1-\varepsilon) \alpha^{t+1} N_{i t+1}^{1-\varepsilon} u\left(c_{i t+1}\right)+(1-\varepsilon) \sum_{s=t+2}^{\infty} \alpha^{s} N_{i s}^{1-\varepsilon} u\left(c_{i s}\right)
\end{aligned}
$$

Substituting

$$
(1-\varepsilon) \sum_{s=t+2}^{\infty} \alpha^{s} N_{i s}^{1-\varepsilon} u\left(c_{i s}\right)=n_{i t+1} \lambda_{i t+1}\left(\beta w_{t+1}+k_{i t+2}\right)
$$

and using (16) again, we find:

$$
\begin{aligned}
& (1-\varepsilon) \alpha N_{i t+1}^{1-\varepsilon} u\left(c_{i t+1}\right)=n_{i t} N_{i t}^{1-\varepsilon} u^{\prime}\left(c_{i t}\right)\left(\beta w_{t}+k_{i t+1}\right) \\
& \quad-\alpha N_{i t+1}^{1-\varepsilon} u^{\prime}\left(c_{i t+1}\right) n_{i t+1}\left(\beta w_{t+1}+k_{i t+2}\right)
\end{aligned}
$$

Replacing $n_{i t}=N_{i t+1} / N_{i t}$ and

$n_{i t+1}\left(\beta w_{t+1}+k_{i t+2}\right)=R_{t+1} k_{i t+1}+w_{t+1}-c_{i t+1}$, we finally obtain the trade-off:

$$
\begin{gathered}
(1-\varepsilon) \alpha u\left(c_{i t+1}\right)=n_{i t}^{\varepsilon} u^{\prime}\left(c_{i t}\right)\left(\beta w_{t}+k_{i t+1}\right) \\
-\alpha u^{\prime}\left(c_{i t+1}\right)\left(R_{t+1} k_{i t+1}+w_{t+1}-c_{i t+1}\right)
\end{gathered}
$$

